\title{
Application of TPA techniques to identify operational forces in optical payloads interfaces
}

Alexandre C. R. Ramos, Leopoldo R. O. Pisanelli

Alexandre C. R. Ramos, Leopoldo R. O. Pisanelli, "Application of TPA techniques to identify operational forces in optical payloads interfaces," Proc. SPIE 11852, International Conference on Space Optics — ICSO 2020, 1185240 (11 June 2021); doi: 10.1117/12.2599802

SPIE Event: International Conference on Space Optics - ICSO 2021, 2021, Online Only 


\section{International Conference on Space Optics-ICSO 2020}

Virtual Conference

30 March-2 April 2021

Edited by Bruno Cugny, Zoran Sodnik, and Nikos Karafolas
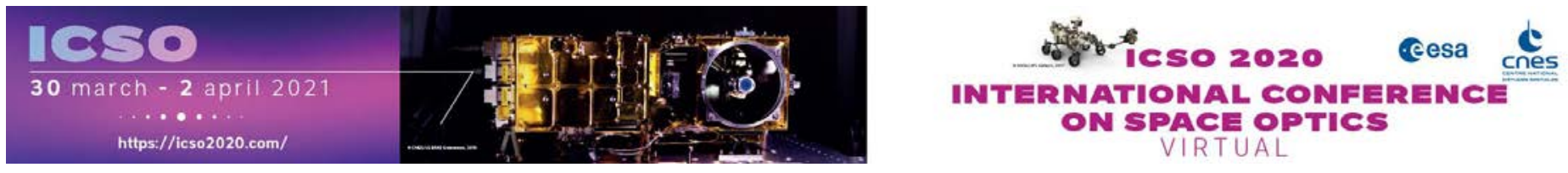

\section{Application of TPA techniques to identify operational forces in optical payloads interfaces}

\section{Cesa issopocesalings lecnes}




\title{
Application of TPA techniques to identify operational forces in optical payloads interfaces
}

\author{
Alexandre C. R. Ramos ${ }^{\mathrm{a}, \mathrm{b}}$, Leopoldo R. O. Pisanelli ${ }^{\mathrm{a}}$ \\ ${ }^{a}$ Laboratory of Dynamics, 400 Trabalhador São Carlense Av., São Carlos, SP, Brazil \\ 13566-590; Dept. of Mechanical Engineering, Univ. of Sao Paulo. \\ ${ }^{\mathrm{b}}$ OPTO Space and Defense, 1071 Joaquim Augusto Ribeiro de Souza St, São Carlos, \\ SP, Brazil, 13563-330.
}

\begin{abstract}
The transfer paths analysis (TPA) is a useful tool to solve noise and vibration problems, since it helps in the study of sources and propagation paths, allowing proposing efficient modifications to minimize vibration in the regions of interest even at early stages in the process of build physical payloads prototypes. In classical TPA, the operational forces are characteristic of the complete system, active subsystem more passive subsystem, this implies that, with each modification in one of the subsystems, it is necessary to redo all tests for the correct determination of noise or vibration at the points of interest of the object under test, constituting one of the obstacles to the widespread use of classical TPA in the product development process. For this reason, in recent years, interest in the development of faster and simpler techniques for analyzing energy transfer paths has been renewed, aiming to bypass the operational limitations of classical TPA. In this scenario, the set of methods called component-based TPA stands out, which is a set of methods that characterizes the excitation of vibration or a noise source through a set of equivalent forces or speeds inherent only to the active subsystem. In this way, the responses, at points of interest located in the passive subsystem, can be calculated using these forces and the FRF of the complete system. The main methods of this class of TPA are the blocked force, freevelocity, hybrid interface and in situ. This work presents a review of component-based TPA methods and proposes the use of these methods in the process of determining equivalent forces in spatial payloads of the active subsystem that, combined with the dynamics of the passive subsystem, allows to predict vibration at points of interest located in the passive subsystem, without having to analyze the complete system.
\end{abstract}

Keywords: TPA, Modular Platform, Payload Structure, Environmental Testing

\section{INTRODUCTION}

In the last decades there has been an increasing demand for the reduction of time and the cost of the development of new products in the space area. This growth in demand increased with the "New Space" philosophy that incorporates new ideas for business models, use of state-of-the-art technologies, additive manufacturing, modularization as well as the willingness to take on higher risks [1]. In the design phase of an optical payload, the requirements and environmental tests in which the instrument will be checked from the qualification model to the flight model are defined, after the optical instrument is validated, it is assembled and integrated in a modular platform that will be submitted to new tests such as vibration tests with lower intensities. In this context, this work seeks to encourage the use of the transfer path analysis (TPA) tools to validate some types of tests such as vibration. The first publications on studies of Transfer Path Analysis were motivated by confidential security demands in the military sector. In the 1950s and 1960s, many publications dealt with topics such as the reduction of noise and vibration transmission from ship and submarine engines, through dynamic absorbers and decoupling mechanisms to minimize the vibro-acoustic transmission between the interfaces of the ships, and make them more stealthy. With the rapid development of airplanes and spacecraft, in the same period, the concepts of TPA began to be used to study problems of fatigue and stability (flutter) due to active vibrations or induced by aerodynamic loads in aircraft. In the last decades, TPA started to be associated with noise, vibration and harshness $(\mathrm{NVH})$ engineering, mainly in the automotive industry, driven by the increasing expectations of customers regarding acoustic and vibratory comfort [2]. The approach is particularly suitable for complex problems, as it allows multiple input and multiple output (MIMO) systems to be divided into discrete excitations and transfer paths, providing excellent vehicle diagnostic information [3]. In the classic TPA model, the system is divided into three parts which consists of the active source, transfer path and 
receiver. The first is the source of vibration, which can be acoustic or structural, called the active subsystem, the second is the propagation paths of the vibro-acoustic energy, for example, fixation interfaces and the last part is the receiver, passive subsystem, which is the target of vibro-acoustic energy which in this work is indicated as the optical payload, as shown in figure 1.

VIBRATION SOURCE

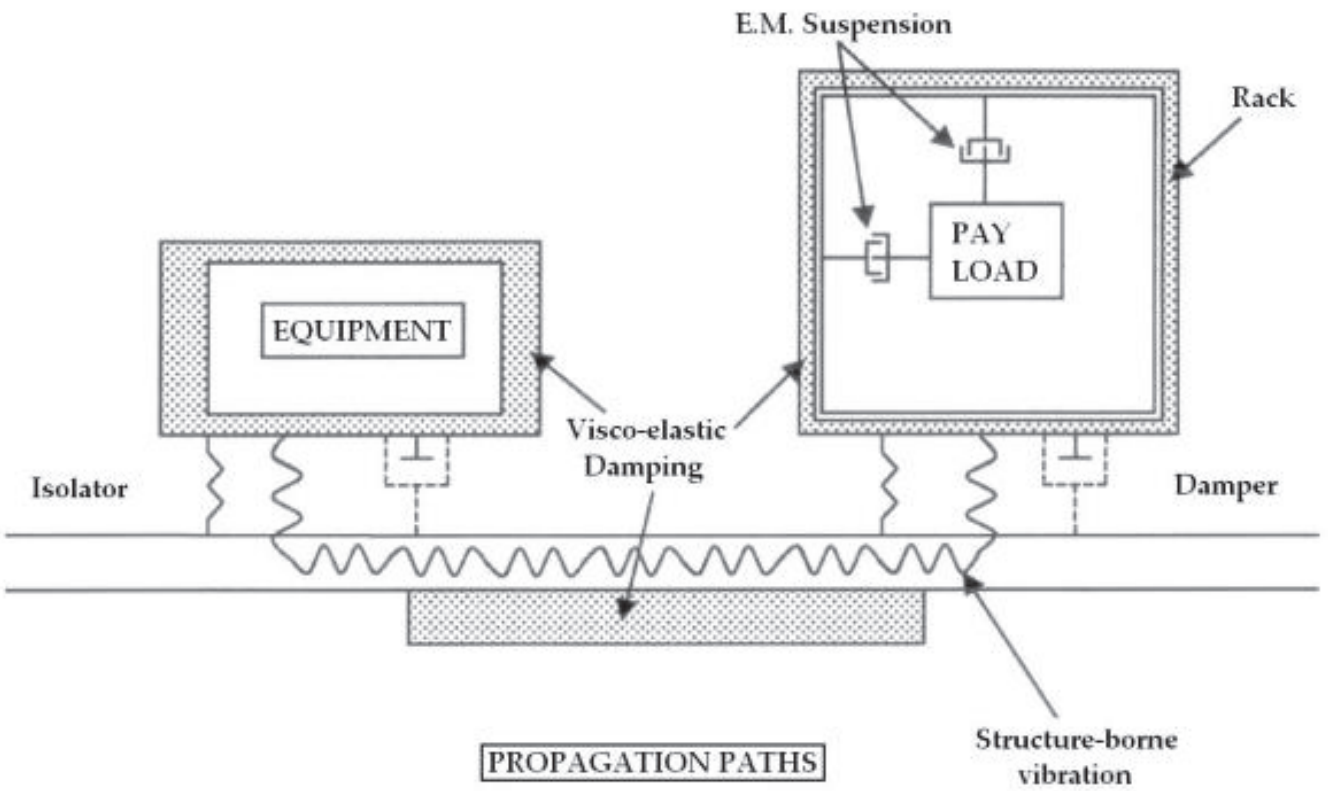

Figure 1. Vibration attenuation, isolation and damping. [4]

With the use of transfer path analysis it is possible to identify which propagation path contributes more to the energy received from an active system in certain frequency ranges, so it is possible to act directly in the path of greater energy transmission, either by using insulators, increased rigidity, mass, among other methods.

In figure 2 below it is possible to observe the classes of TPA and the test flows.

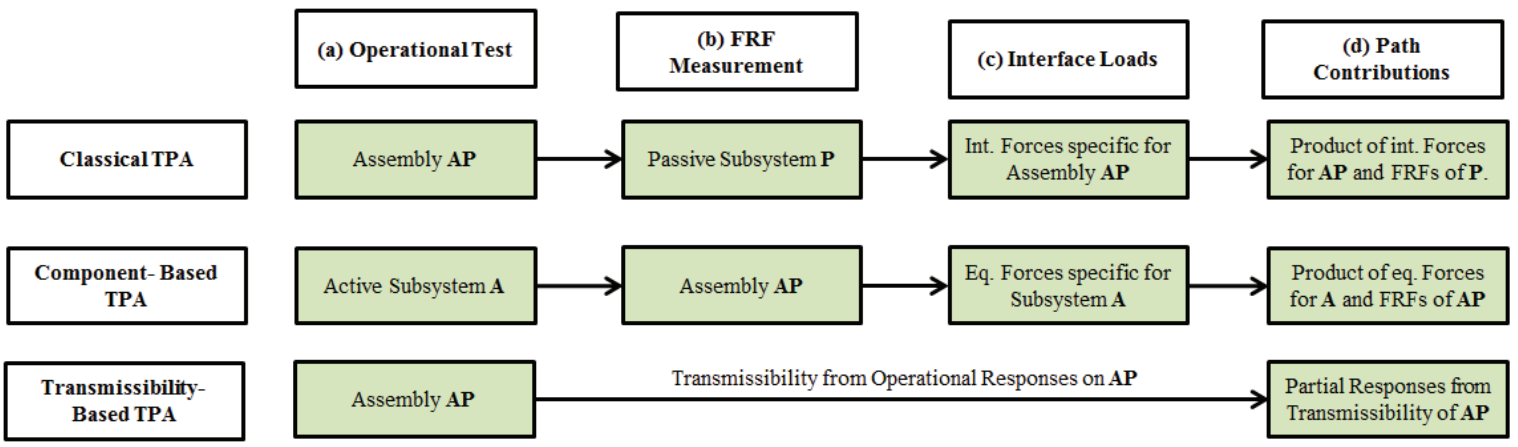

Figure 2. TPA Classes and flows [2] 
TPA Classic methods and those based on TPA transmissibility are used to identify transfer paths in existing projects; component-based TPA methods are valuable in the development stages of the project when the final product or prototype has not yet been released.

In the component-based TPA method, the excitations of the vibration sources are characteristics inherent only in this subsystem, that is, these forces are independent of the dynamic characteristics of the passive system. Thus, this work proposes the use of TPA methods based on components to optimize the development of new products in the space area.

A modular platform has several equipment that generates active vibrations in the satellite structure and can be used as examples of TPA applications based on components, as can be seen in figure 3 . It is possible to identify the energy sources that generate micro vibrations in the satellite payload during its period in orbit. The reaction wheels, for example, can make the images of an optical payload blurry, thus damaging the quality of the images captured by the instrument detectors.

\begin{tabular}{|lcc|}
\hline Source & Harmonic & Transient \\
\hline Reaction wheels & $\mathrm{X}$ & \\
Control Momentum Gyros & $\mathrm{X}$ & \\
Gyroscopes & $\mathrm{X}$ & \\
Solar array drive mechanisms & $(\mathrm{X})$ & $\mathrm{X}$ \\
Antenna pointing mechanisms & & $\mathrm{X}$ \\
Mirror scan mechanisms & $\mathrm{X}$ \\
Cryogenic coolers & & $\mathrm{X}$ \\
Micro-thrusters, gas flow regulators & & $\mathrm{X}$ \\
Latch valve & $\mathrm{X}$ & $\mathrm{X}$ \\
Heat pipe & & $\mathrm{X}$ \\
Relay, RF switch & & $\mathrm{X}$ \\
Sudden stress release & & \\
Clank phenomena & & \\
(e.g. electromagnetic force effects, & & \\
MLI foil buckling) & & \\
\hline
\end{tabular}

Figure 3. Potential classification of micro-vibration disturbance sources [4]. 


\section{THEORY OF COMPONENT BASED TPA}

Component based TPA is a relatively new TPA technique that allows to characterize a source component independently from the receiver structure by a set of blocked forces and to predict its behavior when coupled to different receivers, allowing for a virtual assembly [7].

The system modeling will be performed via the admittance matrices of the assembled system, using the dynamic substructuring approach. The calculation sequence was based on the studies by Seijs et al. [5] [6] and Almiron et al. [8].

Figure 4 below shows the assembly AB representing a multi-mission platform and its payload. The active component of the system will be the reaction wheels, which for simplicity of analysis will be represented by only one source of active excitation. The energy transfer paths, for simplification, will be represented by a dot, and the passive energy receiver will be represented by an element of an optical payload as a mirror for example.

The transfer function between the force $\mathrm{fl}$ of the reaction wheels at position $\mathrm{u} 1$ and the mirrors at position u3, can be represented by Equation 1 below:

$$
u_{3}(w)=Y_{31}^{A B}(w) f_{1}(w)
$$

Where $Y_{31}^{A B}(w)$ represents the frequency response function of the admittance measured in the assembly $\mathrm{AB}$, the frequency will be omitted from the equations for simplicity of notation.
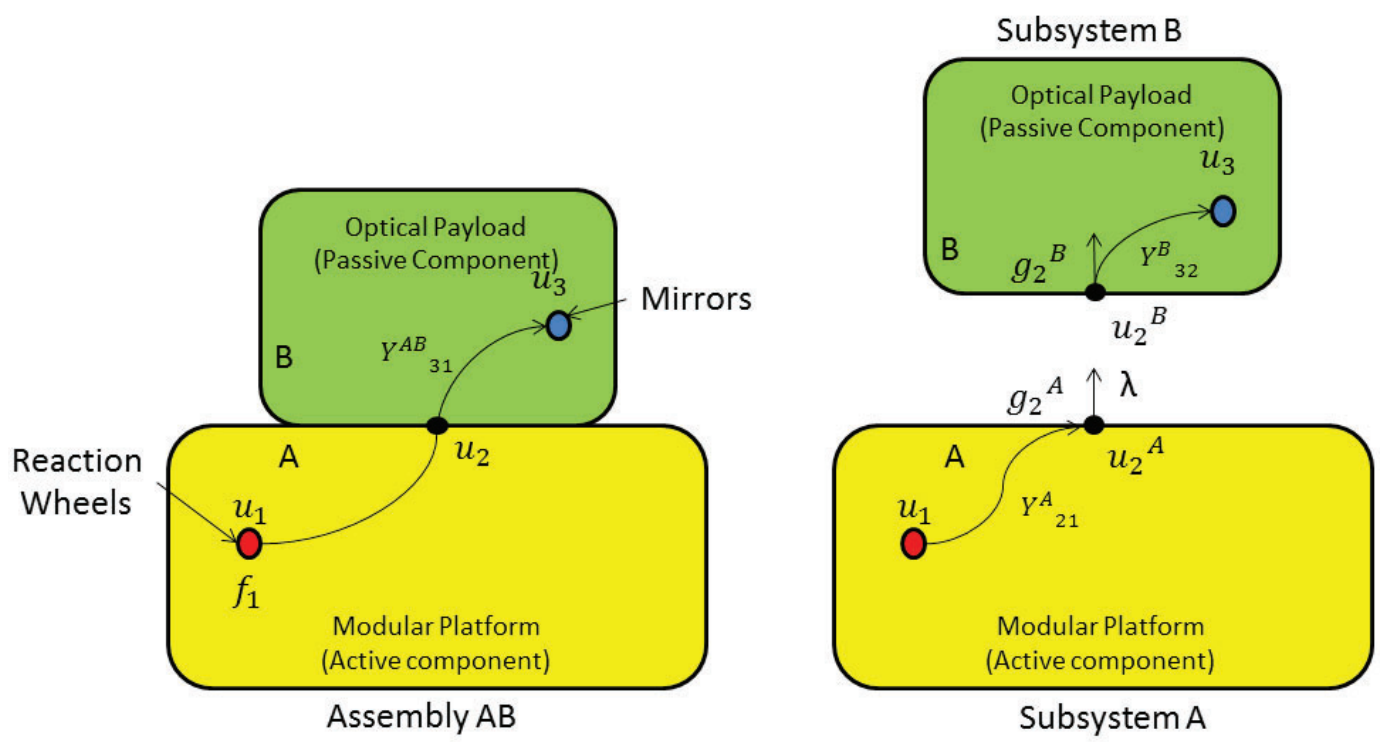

Figure 4. System TPA mounted (4a) and decoupled (4b)

In figure $4 \mathrm{~b}$ we can represent the transfer function of the system by the FRFs and the system equations increased by the forces of unknown interfaces $\mathrm{g} 2$ for the active and passive subsystem. As the system is only excited at node 1 then:

$$
\left[\begin{array}{l}
u_{1} \\
u_{2}^{A} \\
u_{2}^{B} \\
u_{3}
\end{array}\right]=\left[\begin{array}{cccc}
Y_{11}^{A} Y_{12}^{A} & 0 & 0 \\
Y_{21}^{A} Y_{22}^{A} & 0 & 0 \\
0 & 0 & Y_{22}^{B} & Y_{23}^{B} \\
0 & 0 & Y_{32}^{B} Y_{33}^{B}
\end{array}\right]\left[\begin{array}{c}
f_{1} \\
g_{2}^{A} \\
g_{2}^{B} \\
0
\end{array}\right]
$$


The displacements in the interfaces are the same, as seen in Equation 3, and the interface forces have the same magnitude with exchanged signals to maintain the balance of the system, according to Equation 4.

$$
\begin{gathered}
u_{2}^{A}=u_{2}^{B} \\
\lambda=g_{2}^{A}=-g_{2}^{B}
\end{gathered}
$$

Multiplying the second and third lines of Equation 2 and equalizing them, Equation 5 is obtained below:

$$
Y_{21}^{A} f_{1}+Y_{22}^{A} g_{2}^{A}=Y_{22}^{B} g_{2}^{B}
$$

Replacing Equation 4 in Equation 5 and rearranging the terms in Equations 6 and 7, the results are obtained:

$$
\begin{gathered}
\left(Y_{22}^{A}+Y_{22}^{B}\right) \lambda=-Y_{21}^{A} f_{1} \\
\lambda=-\left(Y_{22}^{A}+Y_{22}^{B}\right)^{-1} Y_{21}^{A} f_{1}
\end{gathered}
$$

Substituting Equation 1 in Equation 8, is obtained the Equation 9 which represents the global FRF by coupling the subsystems admittances.

$$
\begin{gathered}
u_{3}=-Y_{32}^{B} \lambda=\left[Y_{32}^{B}\left(Y_{22}^{A}+Y_{22}^{B}\right)^{-1} Y_{21}^{A}\right] f_{1} \\
Y_{31}^{A B}=Y_{32}^{B}\left(Y_{22}^{A}+Y_{22}^{B}\right)^{-1} Y_{21}^{A}
\end{gathered}
$$

\subsection{Response at passive side}

Similar to performed by Equation 1 that relates the source of operational excitation $\mathrm{f} 1$ to the response on the side of the receiver $\mathrm{u} 3$, now looking for an external force equal to $f_{2}^{e q}$ that produces the same response (Figure 5a).

$$
\begin{gathered}
u_{3}=Y_{32}^{A B} f_{2}^{e q}=\left[Y_{32}^{B}\left(Y_{22}^{A}+Y_{22}^{B}\right)^{-1} Y_{22}^{A}\right] f_{2}^{e q} \\
f_{2}^{e q}=\left(Y_{22}^{A}\right)^{-1} Y_{21}^{A} f_{1}
\end{gathered}
$$
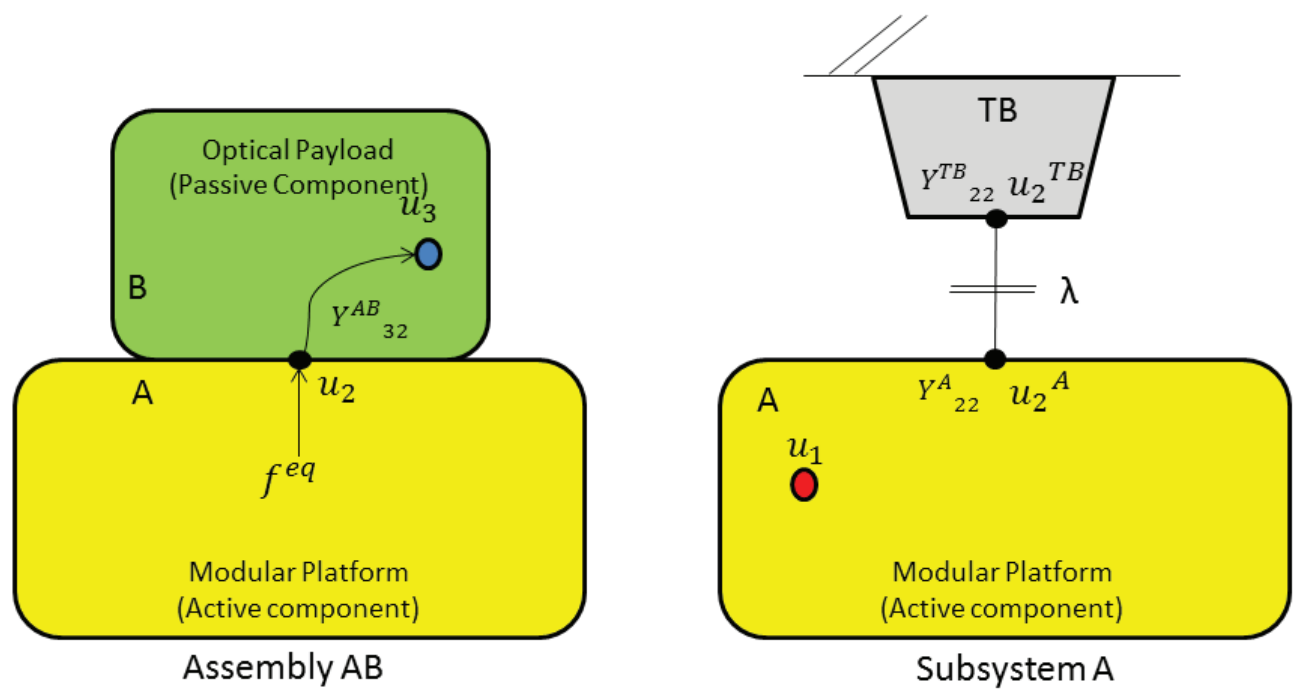

Figure 5. System TPA assembled with equivalent force (5a) and uncoupled in rigid union (5b) 


\subsection{Forces from test bench measurements}

To characterize the excitations of the operational source of the active part, the subsystem is mounted on a test bench (TB) in an extremely rigid manner and with interface connections similar to those found in the platform, as shown in figure $5 \mathrm{~b}$. Thus, the following system of equations can be established for the general case [5][6].

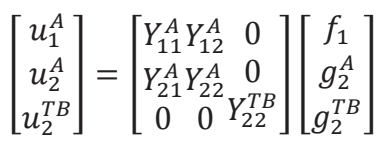

Considering the balance of forces, that $g_{2}^{A}=-g_{2}^{T B}$ and that the system is rigidly coupled, $u_{2}^{A}=u_{2}^{B}$, and considering the operational forces measured with load cells, $g_{2}^{*}$, and the operational displacements measured with accelerometers, $u_{2}^{*}$. Thus, from Equation 12 are obtained the Equations 13 and 14.

$$
\begin{gathered}
g_{2}^{*}=\left(Y_{22}^{A}+Y_{22}^{T B}\right)^{-1} Y_{21}^{A} f_{1} \\
u_{2}^{*}=u_{2}^{A}=\left[I-Y_{22}^{A}\left(Y_{22}^{A}+Y_{22}^{T B}\right)^{-1}\right] Y_{21}^{A} f_{1}
\end{gathered}
$$

\subsection{Blocked interface force}

The blocked force method considers the interface coupling to be extremely rigid, so the value of $Y_{22}^{T B}$ and the displacement $u_{2}^{*}$ are null, so the equivalent force at the interface is given by Equation 17 which in practice are the measurements of load cells. The blocked force method is useful for calculations at low frequencies ranges.

$$
\begin{gathered}
g_{2}^{*}=-\left(Y_{22}^{A}\right)^{-1} Y_{21}^{A} f_{1} \\
u_{2}^{*}=0 \\
f_{2}^{e q}=-g_{2}^{*}
\end{gathered}
$$

\subsection{Free velocity}

Another TPA method derived from Equations 13 and 14 is the free velocity method, in which the active subsystem is in a free-free condition, that is, forces at interfaces are null according to Equation 18, so the equivalent force is given by Equation 20. This method is useful for high frequencies.

$$
\begin{gathered}
g_{2}^{*}=0 \\
u_{2}^{*}=Y_{21}^{A} f_{1} \\
f_{2}^{e q}=\left(Y_{22}^{A}\right)^{-1} u_{2}^{*}
\end{gathered}
$$

\subsection{Hybrid Interface}

Based on the TPA advantages and disadvantages of the blocked force and free velocity methods, the concept of hybrid interface TPA emerges that covers the qualities of the two methods for a wide frequency range, as explained in Equations 21.

$$
f_{2}^{e q}=-g_{2}^{*}+\left(Y_{22}^{A}\right)^{-1} u_{2}^{*}
$$




\subsection{In-situ}

In the in situ method, the active and passive subsystems do not need to be separated in any of the stages of the analysis. The responses at the receivers and indicator points are measured in the operational condition and the FRF measured with the active component turned off, mounted to the passive system and excited externally, for example, via impact hammer or shaker, the equations that determine the In-situ method can be observed in the Eq. 22 and Eq. 23.

$$
\begin{gathered}
f_{2}^{e q}=\left(Y_{22}^{T B}\right)^{-1} u_{2}^{*}+\left(Y_{22}^{A}\right)^{-1} u_{2}^{*} \\
f_{2}^{e q}=\left(Y_{22}^{A T B}\right)^{-1} u_{2}^{*}
\end{gathered}
$$

\section{CONCLUSION}

The use of modern tools in the area of vibrations is an important step towards simplifying qualification tests, especially in space equipment under the new space approach. This paper proposed a summary review of the TPA methods based on components that can be of great value in the development of a new product, mainly in hybrid projects where part of the data is obtained experimentally and the other part by finite element simulations (FEM).

\section{REFERENCES}

[1] Koechel, S., Langer, M., "New Space: Impacts of Innovative Concepts in Satellite Development on the Space Industry", 69th International Astronautical Congress, 2018.

[2] Seijs, M. V., de Klerk, D., Rixen, D. J., "General framework for transfer path analysis: History, theory and classification of techniques", Mech. Syst. Signal Process, 2015.

[3] Elliott., A. S., Moorhouse, A. T.,Huntley, T., Tate, S. "In-situ source path contribution analysis of structure borne road noise", Mech. Syst. Signal Process, 2013.

[4] ECSS-E-HB-32-26A "Space engineering Spacecraft mechanical loads analysis handbook", 19 February 2013, rev A.

[5] Seijs, M. V., Pasma, E. A., de Klerk, D., Rixen, D. J.,“A comparison of two component TPA approachesfor steering gear noise prediction", Proceedings of the 33rd IMAC, 71-79, 2015.

[6] Seijs, M. V., Pasma, E. A., de Klerk, D., Rixen, D. J.,“ A robust Transfer Path Analysis method for steering gear vibrations on a test bench", ISMA2014 International Conference on Noise and Vibration Engineering, 2014.

[7] Bianciardi, F., Almiron, J. O., Risaliti, E., Corbeels, P., "Road Noise assessment using componentbased TPA for a tire assembly", SIA-CTTM 2018 Automotive NVH Comfort, 2018.

[8] Almiron, J. O., Corbeels, P., Bianciardi, F., Desmet, W., "Predicting vibration levels on an experimental test case by using invariant loads (e.g. blocked forces) as source characterization", ISMA 2018 International Conference on Noise and Vibration Engineering, 2018. 